\title{
Ueber die Fähigkeit der Leber, Zucker aus Fett zu bilden.
}

Von

Prof. J. Seegen (Wien).

Die Ernährungsversuche die ich angestellt habe, führten zu dem kaum anfechtbaren Schlusse, dass die Leber unter gewissen Ernährungsbedingungen aus Fett Zucker bildet. Ich habe Thiere durch 7-9 Tage fast ausschliesslich mit Fett gefiittert, es wurden der reichen Fettnahrung nur kleine Mengen Fleisch zugefügt. Das aus der Leber strömende Blut führte ausnahmslos nahezu doppelt so viel Zucker als das in die Leber eingeströmte Blut. Durch directe Beobachtung wurde annährend die Menge des die Leber durchströmenden Blutes bestimmt. Auf Grundlage der in dieser Weise gewonnenen Ziffern, ebenso wie auf Grundlage der von Heidenhain für die Bluteirculation durch die Leber berechneten Ziffern konnte annähernd die Menge Zucker, welche innerhalb einer Zeiteinheit die Leber verlässt, festgestellt werden, und es ergab sich, dass wenn wir die kleinste der gefundenen Ziffern zur Grundlage nahmen, die Zuckerausfuhr bei unseren Versuchsthieren innerhalb 24 Stunden 2-300 gr betragen muss.

Die Stoffe, uiber welche der Körper als Bildungsmatrial verfügt, sind Kohlehydrate, Eiweisskörper und Fett. Die Kohlehydrate entfielen bei der Betrachtung, da diese bei den Fettfütterungsversuchen nicht zugeführt wurden, und die Leber nur sehr geringe Mengen davon enthielt. Damit die Menge des gebildeten Zuckers aus den Albuminaten entstehen könne, mïsste eine beträchtliche Menge derselben umgesetzt werden. Ich hielt einige der Hunde während der ganzen Versuchsdaner im Käfig, sammelte den Harn und bestimmte den Stickstoffgehalt desselben. Dadurch wurde annähernd die Menge der umgesetzten Eiweisskörper resp. des Fleisches bestimmt, und es ergab sich, dass die gesammte Menge der während der ganzen Fütterungsdauer umgesetzten Albuminate nicht ausgereicht baben würde für die Zuckerbildung des inner- 
halb 24 Stunden aus der Leber ausgeführten Zuckers. Es folgte also mit zwingender Nothwendigkeit, dass das Fett ganz oder zum grossen Theile das Material für dic Zuckerbildung geliefert habe. Dasselbe gilt von den Hungerversuchen. Die Kohlehydrate, die beim Beginne der Hungerperiode in der Leber vorhanden sein könnten, würden, selbst wenn, was nicht der Fall war, die reichste Zucker- oder Dextrinfütterung vorangegangen wäre, nicht ausgereicht haben als Material für die Zuckerbildung eines Tages. Ebenso war der Fleischumsatz weleher auf Grundlage des im Harn ausgeschiedenen Stickstoffes festgestellt war weitaus ungenügend für die Bildung des ausgefïhrten Zuckers, es erïbrigt also auch bei den Hungerversuchen nur an Fett als an das vorzüglichste Bildungsmaterial zu denken, was auch mit dem grossen Fettverbrauch während des Hungerns im vollsten Einklange steht.

Die aus den Ernährungsversuchen gewonnenen Thatsachen stellen es also unzweifelhaft fest, das die Leber aus Fett Zucker bildet. Es bedürfte eigentlich gar keines weiteren Beweises, denn diese am lebenden Thiere unter normalen Verhältnissen gewonnene Erfahrung ist beweiskräftig genug. Doch schien es mir von Interesse, ob es gelingen könnte, experimentell die Umbildung von Fett in Zucker durch die Kraft der Leberzelle nachzuweisen und ich habe zu diesem Zwecke eine Reihe von Versuchen angestellt, die ich nachstehend mittheile.

Die Versuche wurden ausschliesslich an $\mathrm{Hunden}$ angestellt und die Versuchsanordnung war folgende:

Das Thier wurde aufgebunden, eine Carotis blossgelegt und aus derselben 2-300 ccm Blut entzogen, welches geschlagen und colirt wurde. Das Thier wurde durch einen Herzstich getödtet, der Bauch geöffnet, die Leber herausgenommen, von derselben je nach Bedarf 2-3 Stiicke zu 40-50gr genommen, sehr fein geschnitten. Die fein geschnittene Masse des einen Stückes wurde mit dem für den Versuch bestimmten Fettkörper und circa 60 $-80 \mathrm{~cm}$ Blut innig gemischt und in eine Flasche mit Drexel'schem Verschluss gegeben. In eine zweite Flasche wurde die gleich grosse Portion Leber mit Blut innig gemischt gegeben, die beiden Flaschen wurden durch ein Kautschukrohr verbunden und in ein Luft- oder Wasserbad mit constanter Temperatur von $35-40^{\circ} \mathrm{C}$. gestellt, die Flasehen mit dem Aspirator verbunden und durch 5-6 Stunden Luft durchgesaugt. Wenn die Versuche mit Seife oder Fettsäuren 
angestellt wurden, musste, weil die Flüssigkeit beim selbst vorsichtigsten Durchleiten der Luft leicht schäumte und dadurch Blut übergerissen wurde, zur Vermeidung dieses Verlustes eine, zuweilen selbst zwei Flaschen zwischen der Versuchsflasche und dem Aspirator eingeschaltet werden.

Das Fett, welches ich benutzte, war fast immer vegetabilisches Fett und zwar in Form einer Emulsion mit Gummi und Wasser hergestellt. Hätte ich bloss flüssiges Fett (Oel) genommen, wäre es in die Höhe gestiegen und hätte sich nie mit der Leber gemischt. Da es jedoch denkbar war, dass auch das Gummi der Emulsion auf die Zuckerbildung einen Einfluss übt, habe ich in dem Controlversuch die gleiche Menge Gummi mit Wasser gemengt der Leber und dem Blut zugefügt. Es wäre mir erwiunscht gewesen das Fett in einer anderen Weise emulgiren zu können, ohne ein Kohlehydrat einzufithren, weil ich sehen wollte ob die Leber ausser Zucker auch Kohlehydrate aus dem Fett zu bilden vermag, was natürlich, wenn Kohlehydrate zugefiugt werden, ausgeschlossen ist aber es gelang mir nicht, and Versuche von Chemikern und Pharmaceuten, die sich zu diesem Zwecke für mich bemühten blieben erfolglos. Reines Saponin wollte ich nicht anwenden, weil es kein indifferenter Körper ist. Die Tinctura Quillaja, die sehr gut emulgirte, zeigte bei der Prïfung beträchtliche reducirende Wirkung anf Kupferoxyd. Heftiges Schütteln mit Wasser wie es Gad angegeben, kann im besten Falle nur minimale Mengen Fett in Emulsion balten. Emulsin war ganz erfolglos - es bildet sich rasch eine Emulsion, die von sehr kurzem Bestande war. Die meisten Oelsamen die beim Auspressen eine schöne Emulsion geben, sind reich an Zucker und an Kohlehydraten. In einigen Versuchen benutzte ich eine Emulsion aus Mohnsamen (sem. pap. alb.), nachdem ich mich überzeugt hatte, dass dieselbe nahezu nicht reducirte.

Nach 5--6 Stunden wurde die Verbindung mit den Aspiratoren gelöst und die in den Flaschen vorhandenen Gemische in Behandlung genommen.

Ich habe hier eine Methode zum erstenmale angewendet, die mir sehr gute Resultate gab und die ich sehr empfehlen kann.

In allen meinen frïheren Versuchen, wo es sich um Zuckerbestimmung in der Leber handelte, habe ich den Zucker in einem alkoholischen Extracte des Decocts bestimmt, und zwar so, dass ich einen Bruchtheil der den Gesammtzucker enthaltenden Decocte durch 
Alkohol fällte, den Niederschlag mit Alkohol wusch, das alkobolische Filtrat einengte und das so eingeengte Filtrat zur Titration mit Fehling'scher Lösung verwendete. Dieser eingeengte alkoh. Extract filtrirt sehr schlecht und trübe. Um dieses zu verhindern muss man während des Eindampfens oft Wasser hinzugeben, wodurch die Fluissigkeit rascher und klarer durchs Filter geht. Das ursprïngliche Decoct ist zur Titrirung gar nicht zu verwenden, man bekommt, wahrscheinlich durch die störenden Eiweisskörper, eine schmutzig gelbe Ausscheidung und kann nie entscheiden, ob die Reduction beendigt ist. Das Fällen durch Alkohol verzögert die Untersuchung; involvirt wahrscheinlich kleine Verluste, da in dem dichten Niederschlage von Kohlehydraten, zumal wenn viel Glycogen vorhanden ist, doch wahrscheinlich Zucker eingeschlossen bleibt. Endlich verursachen die grossen Mengen hochgradigen Alkohols, den man verbraucht, anch beträchtliche Kosten.

Ich mache es nun so, dass ich das der Flasche entnommene Gemisch von Leber, Blut und event. Fett in dem Kochgefässe exwärme und wie bei der Behandlung des Blutes für Znckerbestimmung die Eiweisskörper dureh Eisenchlorid und essigsaures Natron fälle. Wenn das richtige Maass angewendet wurde, geht die Flüssigkeit schon ziemlich klar durch das Filtrirtuch. Die weitere Behandlung ist wie ich sie schon oft angegeben habe; die Coagula, in welchen die Leber eingebettet ist, werden in der Reibschaale verrieben, abermals gekocht (ohne weitere Zuthat von Eisenchlorid) und dieser Prozess so lange wiederholt, bis in der abgepressten Flüssigkeit keine Spur von Zucker nachgewiesen werden kann. Das Decoct wird auf eirca 2-300 cem eingeengt und filtrirt und in diesem Filtrate kann man direct mit der Fehling'schen Lösung den Zucker so schön bestimmen wie in einer Zuckerwasserlösung. Das reducirte rothe Kupferoxydul scheidet sich sehr schön aus und man kann bis auf $1 / 10 \mathrm{ccm}$ genau bestimmen, wann die Reduction beendigt. Ich babe jedesmal 3 Bestimmungen vorgenommen und nicht selten stimmten sie vollkommen, die Variationen bewegten sich innerhalb $1 / 10-2 / 10 \mathrm{ccm}$. Für die Zuckerbestimmung in der Leber ist diese Methode sehr gut, aber sie darf nie angewendet werden, wenn man nebst dem Zucker auch den Glycogengehalt der Leber bestimmen will. Es ist unzweifelhaft, dass durch das Eisenchlorid mindestens ein grosser Theil des Glycogens mit niedergerissen wird, dafür spricht schon, dass die abgepresste Fluissigkeit wenig oder gar nicht opalisirt. 
Landwehr ${ }^{1}$ ) hat die Fällung durch conc. Eisenchloridlösung zur Darstellung und quantitativen Bestimmung des Glycogens benutzt, und giebt an, dass nach seiner Methode auch Glycogen von Dextrin getrennt werden kann, da dieses mit dem Traubenzucker in Lösung bleibt. Ich habe kein Urtheil darüber, in wie weit Nasse's Einwendungen gegen diese ziemlich complicirte Methode der Glycogengewinnung begrïndet sind. Ich habe nur die Erfahrung gemacht, dass die Filtrate der LeberBlutmischungen, die ich in oben angegebener Weise behandelt habe, kein oder nur minimale Mengen Glycogen enthielten, dass aber offenbar ein anderes Kohlehydrat in Lösung blieb, da bei der Erhitzung des Filtrates mit verdünnter Salzsäure in der geschlossenen Röhre die Zuckermenge sich beträchtlich steigerte, was nur durch die Umwandlung jenes Kohlehydrates - wahrscheinlich Dextrin geschehen sein konnte.

Ich habe 10 Versuche mit 10 Thieren angestellt. Die Thiere (Hunde) waren ausnahmslos durch einige Tage (von 3-8 Tagen) mit Fleisch gefüttert, so dass sie wohl und kräftig zum Versuche kamen. Die Versuche verliefen alle in gleicher Weise, nur blieb das Blut nicht bei allen Versuchen arteriell. Zuweilen wurde dasselbe schon nach 1-2 Stunden dunkel. Es mag dies damit zusammenhängen, dass auch die Aspiration nicht stets gleichmässig. von Statten ging. Leberstiickchen verstopften oft die Röhre, so dass Nachhülfe nöthig war.

Da es zwecklos wäre jeden einzelnen Versuch detaillirt mitzutheilen, fasse ich die gewonnenen Resultate tabellarisch zusammen. Ich habe in der letzten Columne der Tabelle die Differenz im Zuckergehalt zwischen den mit und ohne Fett behandelten Leberstiicken verzeichnet. Es giebt diese in Procenten ausgedrickte Differenz nur eine annähernde Vorstellung von der Zuckerzunahme, da die percentische Differenz sich grösser oder kleiner gestaltet, je nach dem Zuckergehalt des Controlstückes und eine Zuckerzunahme von $1,2 \%$ wie in den Versuchen I und II einmal eine percentische Differenz von $35 \%$ und das zweitemal eine solche von $75 \%$ ergiebt, aber man kommt doch dadurch, zumal wenn man eine ganze Reihe von Versuchen überblickt und auch noch das Mittel aus den Differenzen zieht, zu einer richtigen Vorstellung iber die Versehiedenartigkeit der Zuckerbildung in den beiden Versuchsstücken.

1) Zeitschrift für physiol. Chemie 8. 
Ueber die Fähigkeit der Leber Zucker aus Fett zu bilden.

Tabelle.

\begin{tabular}{|c|c|c|c|c|c|}
\hline \multirow{2}{*}{$\begin{array}{l}\text { Versuchs- } \\
\text { nummer }\end{array}$} & \multirow{2}{*}{$\begin{array}{l}\text { Ohne Fett. } \\
\text { Zucker in } \%\end{array}$} & \multicolumn{2}{|c|}{ Mit Fett. } & \multicolumn{2}{|c|}{$\begin{array}{l}\text { Differenz im Zucker- } \\
\text { gehalt. }\end{array}$} \\
\hline & & $\begin{array}{l}\text { Art des } \\
\text { Fettes }\end{array}$ & $\begin{array}{c}\text { Zucker } \\
\text { in } \%\end{array}$ & absolut & relativ in $\%$ \\
\hline I & 3,4 & Olivenöl & 4,6 & $+1,2$ & 35 \\
\hline II & 1,6 & " & 2,8 & $+1,2$ & 75 \\
\hline III & 1,4 & Ricinusöl & 2,3 & $+0,9$ & 64 \\
\hline IV & 2,5 & Mandelöl & 3,0 & $+0,5$ & 20 \\
\hline V & 3,5 & Olivenöl & 4,6 & $+1,1$ & 31 \\
\hline VI & 3,5 & $n$ & 5,0 & $+1,5$ & 42 \\
\hline VII & 3,1 & Leberthran & 3,4 & $+0,3$ & 10 \\
\hline VIII & 1,3 & Mohnöl & 2,5 & $+1,2$ & 92 \\
\hline IX & 2.0 & $"$ & 3,4 & $+1,4$ & 70 \\
\hline $\mathrm{X}$ & 2,2 & $"$ & 3,0 & $+0,8$ & 36 \\
\hline
\end{tabular}

Mittel $\quad 47,5 \%$

Bemerken möchte ich noch, dass ich wiederholt die Gährungsprobe machte, die Gährung geht durch 8-10 Stunden rasch, dann äusserst langsam so dass sie auch nach 8 Tagen nicht abgeschlossen ist. Aber auch die Gährungsresultate ergeben bedeutende Unterschiede im Zuckergehalte; so waren im Versuche VIII die Gährungsresultate: ohne Fett $1 \%$, mit Fett $2 \%$ und im Versuche IX ohne Fett 1,9\%, mit Fett 2,8\%.

Aus diesen Versuchen geht hervor, dass die mit Fett behandelte Leber ausnahmslos mehr Zucker enthält als das in gleicher Weise mit Ausschluss von Fett behandelte Controlstiick, die Zuckerzunahme ist meist eine sehr beträchtliche, sie ist nur in einem Falle in dem mit Leberthran behandelten gering, im Durchschnitte aus 10 Versuchen beträgt die Zunahme nahezu 50\%. Aus diesen Versuchen geht zweifellos hervor, dass dieser mehrgebildete Zucker aus Fett entstanden ist.

Es war von grossem Interesse zu wissen, welcher Bestandtheil des Fettes sich an der Zuckerbildung betheilige, und 
ich habe zunächst einige Versuehe gemacht, in welehen ich die Bestandtheile der Fett bildenden Glyceride getrennt mit der Leber in Verbindung brachte und zwar wurden 3 Versuche angestellt, in welchen statt des Fettes Glycerin benützt wurde. Fuir andere Versuche benutzte ich die aus Schweineschmalz dargestellten Fettsäuren und die aus diesen Fettsäuren dargestellten Seifen. Die Fettsäuren hatten ihren Schmelzpunkt bei $36^{\circ}$, sie wurden mit der Leber verrieben und da das Luftbad $35-40^{\circ}$ hatte, war anzunehmen, dass sie verflüssigt blieben. Ich wendete für einen Versuch 5 gr Fettsäure an. Von den Seifen, welche circa 30 gr Fettsäuren enthielten, benutzte ich circa $15 \mathrm{gr}$, die ich mit dem Blute und der Leber verrieb.

Nachstehende Tabelle giebt die erhaltenen Resultate.

Tabelle.

\begin{tabular}{|c|c|c|c|c|c|}
\hline \multirow{2}{*}{$\begin{array}{l}\text { Versuchs- } \\
\text { nummer }\end{array}$} & \multirow{2}{*}{$\begin{array}{l}\text { Ohne Fett. } \\
\text { Zucker in } \%\end{array}$} & \multicolumn{2}{|c|}{ Mit Fettbestandtheilen. } & \multicolumn{2}{|c|}{$\begin{array}{c}\text { Differenz im Zucker- } \\
\text { gehal }\end{array}$} \\
\hline & & \begin{tabular}{|} 
Art des Fett- \\
bestandtheils
\end{tabular} & $\begin{array}{l}\text { Zucker } \\
\text { in } \%\end{array}$ & absolut & relativ In $\%$ \\
\hline $\mathrm{XI}$ & 3,1 & Glycerin & 3,6 & $+0,5$ & 16 \\
\hline$x^{\top}$ & 3,3 & $"$ & 4,1 & $+0,8$ & 24 \\
\hline XIII & 1,3 & $"$ & 2,1 & $+0,8$ & 61 \\
\hline $\mathrm{XIV}$ & 1,3 & Seife & 2,5 & $+1,2$ & 92 \\
\hline$X V$ & 2,2 & $"$ & 3,3 & $+1,1$ & 50 \\
\hline XVI & 2,5 & $"$ & 3,3 & $+0,8$ & 32 \\
\hline XVII & 2,5 & $\eta$ & 3,2 & $+0,7$ & 28 \\
\hline XVIII & 4,5 & Fettsäure & 4,7 & +0.9 & 08 \\
\hline$X I X$ & 2,7 & $"$ & 3,6 & $+0,9$ & 33 \\
\hline$X X$ & 3,0 & $"$ & 3,7 & $+0,7$ & 23 \\
\hline
\end{tabular}

Also auch bei Behandlung der Leber mit einzelnen Fettbestandtheilen, sowohl mit Glycerin wie mit verseiften Fettsäuren war die Zuckerbildung beträchtlich vermehrt. Am wenigsten constant sind die Versuche mit Fettsäuren, in 2 Versuchen XIX und 
$\mathrm{XX}$ ist ein beträchtliches Zuckerplus, während im Versuche XVIII die Zunahme kaum bemerkenswerth ist.

Es ist zu berïcksichtigen, dass bei den Versuchen mit Fettsäure die ganze Mischung saver wurde, während dieselbe sonst wie die frische Leber selbst nahezu neutral war, dass ferner an eine Lösung derselben im Blute nicht zu denken war und eine Verflüssigung nur so lange bestand, als die Temperatur im Luftbade genïgend hoch war. Die Versuche mit Glycerin wie mit den verseiften Fettsäuren weisen aber unzweifehaft darauf hin, dass beide Fettbestandtheile sich an der Zuckerbildung betheiligen.

Ich habe bei den Versuchen mit Fettbestandtheilen auch das Verbältniss der Gesammtkohlehydrate zu dem des Controlstiickes bestimmt, und zwar indem ich $10-20 \mathrm{ccm}$ des Decocts mit 2-4 ccm 10\% iger Salzsäure in der zugeschmolzenen Glasröhre 10-12 Stunden im kochenden Wasserbade liess. Die eingeschlossene Flüssigkeit blieb ganz klar, nur bei dem Seifendecocte wurde sie dunkel, doch war die Zuckerbestimmung sehr genau ausfïbrbar.

Das Decoct aus der mit Fettbestandtheilen behandelten Leber enthi elt ausnahmslos mehr Kohlehydrate (nach Abzug des Zuckers) als das Decoct aus dem Controlstiicke, was soviel sagen wiirde, dass aus den Fettbestandtheilen nebst dem Zucker auch andere Koblehydrate, und speciell Dextrin gebildet wurden. Doch kann ich den gewonnenen Ziffern nicht volle Beweiskraft für diese Thatsache beilegen, da es vorerst sicher gestellt werden müsste, dass sämmtliches Glycogen dureh die Behandlung mit Eisenchlorid ausgefällt wurde und dass kein anderes Kohlehydrat mitgerissen werde, das ungleichmässige Zurückbleiben des einen oder des andern Kohlehydrats könnte sonst die Differenz in dem Befunde veranlasst haben, wiewohl es immer merkwürdig bliebe, dass das minus immer auf Seite des Controlstiickes gefunden wurde.

Wie die Umwandlung ron Fett in Zucker zu Stande kommt ist uns vorläufig vollständig unbekannt. In jedem Falle müssen wir uns denken, dass noch eine beträchtliche Menge Sauerstoff hinzutreten muss, wenn auch nur einem grösseren Bruchtbeile des im Fette enthaltenen Kohlenstoff entsp rechend, Zucker entstehen soll.

Sehr fördernd für unsere Einsicht in die sich in der Leber abspielenden Prozesse wäre es, den Sauerstoff des in die Leber einströmenden wie den des aus der Leber ausströmenden Blutes 
kennen za lernen. In einem Referate ${ }^{1}$ ) ïber eine von G. Kempner ausgeführten Arbeit "Ueber den Einfluss des Sauerstoffgehaltes der Einathmungsluft auf den Ablauf der Oxydationsprocesse" finde ich die Bemerkung, dass das mittlere Venenblut noch recht ansehnliche Mengen von Sauerstoff enthält, dass aber aus gewissen Organen speciell aus der Leber das Blut bereits normaler Weise nahezu sauerstofffrei abfliesse. Nähere Daten fehlen und es. wäre gewiss lohnend, Gasanalysen mit Pfortader- und Lebervenenblut anzustellen.

Es ist gewiss von grösstem Interesse, dass die Zuckerbildung aus Fett, die für den thierisehen Organismus dureh meine Fïtterungsversuche wie durch das directe Experiment zuerst erkannt wurde, als ein normaler Process im Entwicklungsleben vieler Pflanzen längst gekannt ist. Sachs ${ }^{2}$ ) hat im Jahre 1859 zuerst nachgewiesen, dass bei der Keimung fetthaltiger Samen auf Kosten des Fettes Stärke und Zucker gebildet wird. „In dieser wie ich glaube völlig neven Thatsache, so spricht sich Sacbs aus, liegt sowohl in chemischer wie in physiologischer Beziehung viel Ueberraschendes." Heute ist diese Thatsache von allen Botanikern anerkannt und wird durch ein einfaches sehr hiibsches Schulexperiment constatirt. In einem aus Oelsamen im Dunklen gezogenen Keimling werden die Cotyledonen dürch Jodtinktur tief blau gefärbt. Das Fett des Samens ist verschwunden und in dem Keime, speciell in den Cotyledonen, bat sich Stärke angehäuft ${ }^{3}$ ).

1) Maly's Jahresbericht. Bd. XIV. S. 349.

2) Sachs, Ueber Bildung von Stärke bei der Keimung fetthaltiger Samen. Bot. Zeitung. 1859, p. 178.

3) Prof. Wiesner, der Vorstand des Wiener Instituts für Pflanzenphysiologie, hatte die Güte mir dieses hübsche Experiment an Keimlingen, die aus Rettigsamen gezogen waren, zu zeigen.

Zugleich machte er mich auf einen andern sehr interessanten Versuch aufmerksam, der die Art der Umwandlung des Fettes boim Keimen fetthaltiger Samen demonstrirt, und als Analogie für ähnliche Vorgänge im Thierkörper von höchster Bedeutung sein dürfte. Lässt man Stärkemehlhaltige Samen unter einer durch Quecksilber abgeschlossenen Glasröhre keimen, tritt keine Veränderung des Gasvolums auf, während bei der Keimung ölhaltiger Samen eine durch das Steigen des Quecksilbers wahrnehmbare Gasreduction eintritt. Die Verminderung des Gasvolums entspricht der Sauerstoffresorption, welche zur Umwandlung des Fettes in Stärke er. forderlich war. 
Hoffentlich wird anch die Thatsache, dass die Leber aus Fett Zucker bildet, die beute noch Chemiker wie Physiologen iiberraschen dürfte, bald allgemein anerkannt werden. Diese Thatsache hat nach mehrerer Richtung Bedeutung: 1. Wir bekommen dadurch noch tiefere Einsicht in die grosse Bedentnng, welche die Leber für den Stoffumsatz hat. Die Leber scheint das grosse Laboratorium zu sein, in welchem die Nahrungsmittel für die Zwecke des Lebens, für Arbeitsleistung und für Wärmebildung umgewandelt werden. Der Prozess, durch welchen dies geschieht ist die Zuckerbildnng. Ich habe schon früher durch directe Versuche bewiesen, dass die Leber aus Pepton Zucker bildet, die Ernährungsversuche die reiche Zuckerbildung bei ausschliesslicher Fleischnahrung, geben die praktischen Belege für die im Kleinen ausgefïhrten, Experimente.

Durch die neu gewonnene Thatsache, dass die Leber aus Fett Zucker bereiten kann und durch die Erfahrung, dass bei ausschliesslicher Fettfiitterung die reiche Zuckerbildung in der Leber fortbesteht, sehen wir die Wirkungssphäre der Leber in Verwerthung der Nahrungsmittel bedeutend erweitert.

Diese Thatsache wird eine noch grössere Bedentung gewinnen, wenn es, wie zu erwarten ist, festgestellt wird was bis jetzt nul Vermuthung ist, dass aus dem Leberglyeogen Fett wird. Die directe Zuckerbildung aus Glycogen findet nicht statt, es ist aber wahrscheinlich, dass das aus den Kohlehydraten in der Leber gebildete Glycogen in Fett und dann in Zucker umgewandelt wird. Auch dieser Prozess hätte seine Analogie in der Pflanzenwelt. Die fetthaltigen Samen, aus deren Fett bei der Keimung Zucker entsteht, enthalten vor der Reife kein Fett, sondern ausschliesslich Stärke und Zucker. Man kann nach Sachs') unreife Samen z. B. von Paeonia von der Mutterpflanze ablösen und sie in feuchter Luft liegen lassen, um dann zu finden, dass die Stärke in ihnen verschwunden and durch fettes Oel ersetzt worden ist.

2. Die Thatsache, dass die Leber aus Fett Zucker zu bilden vermag hat eine biologische Bedeutung, weil dadurch nachgewiesen wird, dass ein sehr wichtiger Stoffwechselvorgang, den man bis jetzt als den Pflanzen eigenthümlich angesehen, der gesammten

1) Sachs, Vorlesungen über Pflanzenphysiologie. 1882. 
$142 \mathrm{~J}$. Seegen: Ueber die Fähigkeit der Leber Zucker aus Fett zu bilden.

organischen Natur gemeinsam ist und dadurch wieder eine Kluft zwischen Pflanzen- und Thierreich ausgefillt wird.

3. Die Kenntniss von der Zuckerbildung aus Fett hat aber auch unzweifelhaft eine wichtige praktische Bedeutung, wir lernen dadurch den vollen Werth des Fettes als Nabrungsmittel kennen. Wenn es sich herausstellt, dass Zucker das eigentliche Brennmaterial des Körpers, seine Kraftquelle für Arbeitsleistung und Wärmebildung ist, dann wird jenes Material für die Erfüllung dieser Lebensaufgaben am werthvollsten sein, aus welchem die grössere Menge Zucker gebildet werden kann. Denken wir, es würde der gesammte Kohlenstoff des Fettes für die Zuckerbildung verwendet, dann würde aus cirea $52 \mathrm{gr}$ Fett $100 \mathrm{gr}$ Zucker gebildet, während $300 \mathrm{gr}$ Fleisch erforderlich wären, um die gleiche Menge Zucker zu bilden, abgesehen davon, dass noch ein Theil des $\mathrm{C}$ des Fleisches zur Bildung von dem dem Körper gar nicht zu Statten kommenden Harnstoff verwendet wird. Die Erfahrung, welche der Wissenschaft oft voraus eilt, hat es längst gefunden, dass wenn es sich um grosse Arbeitsleistung handelt oder um rasche Wärmeerzeugung, ein Stiick Speck oder eine Portion Thran ausgiebiger sind als ein Stück Fleisch. Ernährungsversuche haben gelehrt, dass bei reichem Fettzusatz der Körper nur wenig Fleisch braucht, um auf seinem Bestande zu bleiben. Alle diese Erfahrungen diirften von dem nea gewonnenen Standpunkte ihre Erklärung finden, und die wichtigsten practiseh diätetischen Fragen, wie die Feststellung des Kostmaasses für grosse Gemeinschaften, für verschiedene Arbeitsleistungen, bei verschiedenen Krankeitsformen etc. werden in Zukunft mit diesem wichtigsten Factor des Stoffumsatzes, mit dem Vermögen der Leber, aus Fett Zucker zu bilden, rechnen müssen. 